\title{
Role and Outcome of Endovascular Management for Surgical High Risk Patients with TASC II D Femoropopliteal occlusive Disease: A Single Center Study
}

\author{
Amr Nabil Kamel MD, MSc; Mohamed Mahmoud Zaki, MD, MRCS, MSc; Amr Abdelghaffar \\ Hanfy Mahmoud, MSc \\ Department of Vascular Surgery, Faculty of Medicine, Ain Shams University, Egypt
}

Background: Peripheral arterial disease (PAD) is a medical condition of growing magnitude that usually results from atherosclerosis and subintimal cholesterol deposition leading to stenosis or occlusion in the major arteries supplying the lower extremities. Worldwide prevalence of PAD is estimated to be around $10 \%$. In general, bypass surgery is better adopted for long SFA occlusive lesions of more than $25 \mathrm{~cm}$ length. Using an autologous vein graft is generally more preferable, especially whenever the patient's general condition permits, provided that the patient's life expectancy is more than 2 years. Endovascular treatment is an attractive alternative to open surgical procedures for PAD.

Aim of the work: This prospective cohort study aimed at detecting the role of endovascular management for surgical high risk patients with TASC II D femoro-popliteal occlusive diseases. The primary end point was limb salvage, and secondary end points included amputation free survival, binary restenosis.

Patients and methods: This is a prospective non randomized cohort study which included 54 surgical high risk patients with TASC II D femoro-popliteal occlusive diseases. It is a single arm clinical study with no comparison with open surgery result. The protocol was approved by the local ethics committee of Ain Shams University hospitals and all patients signed an informed consent form.

Results: 54 patients were included: 14 men (25.9\%) and 40 women (74.1\%). Technical success was reported in $48 / 54$ cases ( $88.9 \%$ ), and 6 cases failed (5 received bypass, 1 underwent major amputation). The primary patency within 2 years was $70.9 \%$, assisted primary patency was $73.33 \%$ and secondary patency was $68.18 \%$.

Conclusion: Endovascular management has an effective role in the treatment of TASC II D femero-popliteal occlusive diseases in surgical high risk patients and the limb salvage. Drug eluting balloons have lower incidence of restenosis and need for intervention in comparison to standard balloon. Stent insertion is associated with high incidence of re-intervention in comparison to balloon only angioplasty.

Keywords: Endovascular management, surgical high risk patients, TASC II D femoropopliteal occlusive Disease.

\begin{abstract}
Introduction
Peripheral arterial disease (PAD) is a medical condition of growing magnitude that usually results from atherosclerosis and subintimal cholesterol deposition leading to stenosis or occlusion in the major arteries supplying the lower extremities. Worldwide prevalence of PAD is estimated to be around $10 \% .^{1}$ The vast majority of people with PAD are either symptom free, or do not have any significant functional limitation. ${ }^{2}$ Many people with PAD, however, have limited walking distances, to the extent that might interfere with their quality of life. In addition to the limited walking ability, people with advanced PAD/critical limb ischemia are at an increasing risk for limb loss, reaching 10-40\% at 6 months. $^{3}$ In addition to involving the limbs, PAD is a "manifestation of systemic atherosclerosis that involves other major circulation, such as the cerebral and coronary arteries". The diagnosis of PAD is associated by an $20 \%$ mortality rate at 6
\end{abstract}

months and $50 \%$ at 5 years. ${ }^{3}$ Patients diagnosed with PAD are also estimated to have a 2 to 3 fold increased risk of developing myocardial infarction and cerebrovascular strokes as compared to age and sex matched individuals without PAD. ${ }^{4}$

Endovascular treatment is an attractive alternative to open surgical procedures for peripheral arterial disease (PAD) due to the less procedural risk resulted by it. Numerous therapeutic strategies can be adopted to treat long Femoro-popliteal (FP) lesions, including self-expandable stent, drug eluting balloon or stent, or covered stent. ${ }^{5}$

BASIL trial demonstrated that at 2 years, no difference in overall survival or amputation-free survival by intention-to-treat assignment between surgical intervention and angioplasty in severe limb ischemia. ${ }^{6}$ However, beyond 2 years, patients initially randomized to open bypass surgery had 
superior clinical outcomes compared to those initially randomized to angioplasty. ${ }^{7}$ Consequently, the concluded recommendations from the BASIL trial acknowledged that in patients who are not at high risk for surgical intervention, bypass is recommended for long SFA lesions of more than 25 $\mathrm{cm}$, when an autologous vein is available provided that life expectancy is $>2$ years. $^{7}$

\begin{abstract}
Aim of the work
This is a prospective non randomized cohort study, which is a single arm clinical study to detect the role of endovascular management for surgical high risk patients with TASC II D femoro-popliteal occlusive diseases. Primary end point is limb salvage. Secondary end points include: Amputation free survival, binary restenosis (peak velocity ratio of 2.4 of target lesion or $50 \%$ re obstruction of target lesion), target limb revascularisation (frequency for need for redo procedure (endovascular or open) after successful first procedure due to problem arising from the SAME lesion). Research Question: What is the outcome of Endovascular management in surgical high risk Patients with TASC II D femoro-popliteal occlusive disease with respect to limb salvage? Research Hypothesis: Endovascular treatment of TASC II D femoro-popliteal artery occlusion has a high technical success rate and has an effective role in preventing and delaying amputation.
\end{abstract}

\section{Patients and methods}

This is a prospective non randomized cohort study. Which is a single arm clinical study to detect the role of endovascular management for surgical high risk patients with TASC II D femoro-popliteal occlusive diseases. The protocol was approved by local ethics committee of Ain Shams University hospitals and all patients signed an informed consent form. The primary end point is limb salvage, and secondary end points include amputation free survival, binary restenosis (peak velocity ratio of 2.4 of target lesion or $50 \%$ re obstruction of target lesion) and target limb revascularisation (frequency for need for redo procedure (endovascular or open) after successful first procedure due to problem arising from the SAME lesion).

- Study design: This is a prospective non randomized cohort study.

- Study Population: Surgical High risk patients with critical limb ischemia as defined by Rutherford classification having TASC II D lesions of femoro-popliteal arterial segment.

- Sample size: Total number of patients is 54 cases.

- Study venue: Ain Shams University hospitals (Demerdash \& Ain Shams University Specialized Hospital [ASUSH]).

\section{Inclusion criteria for diagnosing of CLI: 1) Clinical diagnosis:}

- Adult patient with history of critical limb ischemia as defined by Rutherford classification: rest pain (category 4), ischemic ulceration that is not exceeding boundaries of the toes (category 5) \& severe ischemic ulcers or established gangrene (Rutherford category 6 ).

- High risk patients: ASA 3 or ASA 4.

- Intact femoral pulsation / patent Iliac arteries

2) Radiological diagnosis:

- Patients included in the study who had subcategory of TASC D femoropopliteal arterial lesions detected radiologically by duplex $+/-\mathrm{Ct}$ angiography (Chronic total occlusions of SFA $>20 \mathrm{~cm}$ ) or popliteal artery occlusion.

3) Written and signed consent obtained from the patient.

\section{Exclusion criteria:}

- Claudicants or asymptomatic lesions (Rutherford 1, 2, 3).

- Severe diabetic foot infection that render the limb unsalvageable.

- Patients who underwent previous interventions (femoro-popliteal lesion at the target limb).

- Acute ischemia.

- Patient refusal to participate.

- Pregnancy.

- Known allergy to contrast or antithrombotic medications.

- Life expectancy $<3$ months.

\section{Statistical analysis:}

Data was analyzed using SPSS $\subseteq$ Statistics version 24 (SPSS $($ ) Corp., Armonk, NY, USA). The non categorical variables were presented in the form of mean and standard deviation. The categorical variables were presented as percentages. The demographic data and the comorbidities of the patients were related to the number of participating patients, whereas the patency data were calculated according to the number of included limbs.

\section{Results}

This was a prospective non randomized cohort study to assess the role of endovascular management for surgical high risk patients with TASC II D femoropopliteal occlusive diseases. 54 patients were included: 14 men $(25.9 \%)$ and 40 women $(74.1 \%)$ range of age was between 35-87 years with mean of 62 (SD; 10.468). There were $42 / 54$ patients $(77.8 \%)$ underwent Contralateral retrograde access at CFA, 7/54 patients (13\%) Ipsilateral ante grade access at CFA, 5/54 patients (9.3\%) Ipsilateral Retrograde access at popliteal artery. The lesion site was chronic total occlusion (CTO) of superfischial femoral artery : $(48 / 54 ; 88.9 \%)$, popliteal artery $(2 / 54 ; 3.7 \%)$ or both $(4 / 54 ; 7.4 \%)$, (Figure 1 ). 
Cardiovascular risk factors included smoking (31/54; $57.4 \%$ ) the duration of smoking was between 5 to 50 years with mean of 21.47 (SD;11.02), hypertension (41/54; 75.9\%), Diabetes Mellitus (46/54; 85.2\%) in which insulin dependent DM (25/46; 51\%) while non-insulin dependent DM (24/46; 49\%), dyslipidemia (25/54; 46.3\%), and coronary heart disease $(24 / 54 ; 44.4 \%)$, cerebral vascular accident (3/54; 5.6\%), renal impairment $(1 / 54 ; 1.9 \%)$. The mean occlusion length was 20.7 +/- 10 CM (SD; 3.457), (Table 1).

Table 1: Epidemiology of patients

\begin{tabular}{|c|c|c|c|}
\hline & Variable & Number & Valid Percent \% \\
\hline \multirow{2}{*}{ Gender } & Male & 14 & $25.9 \%$ \\
\hline & Female & 40 & $74.1 \%$ \\
\hline \multirow{2}{*}{ Current Smoker } & Yes & 31 & $42.6 \%$ \\
\hline & No & 23 & $57.4 \%$ \\
\hline \multirow{2}{*}{ EX-smoker } & Yes & 36 & $66.7 \%$ \\
\hline & No & 18 & $33.3 \%$ \\
\hline \multirow{2}{*}{ Type of DM } & IDDM & 25 & $51 \%$ \\
\hline & NIDDM & 24 & $49 \%$ \\
\hline \multirow{8}{*}{ Co-morbidities } & CVA & 3 & $5.6 \%$ \\
\hline & DM & 46 & $85.2 \%$ \\
\hline & HTN & 41 & $75.9 \%$ \\
\hline & ISHD & 24 & $44.4 \%$ \\
\hline & $\mathrm{CCF}$ & 3 & $5.6 \%$ \\
\hline & CLD & 1 & $1.9 \%$ \\
\hline & Dyslipidemia & 25 & $46.3 \%$ \\
\hline & Renal Impairment & 1 & $1.9 \%$ \\
\hline
\end{tabular}

Technical success was reported in $48 / 54$ cases $(88.9 \%)$, and 6 cases failed (5 received bypass, 1 underwent major amputation).

Follow-up: The first post-intervention check were scheduled at 2 weeks after the procedure, then at $1,6,9,12,+/-24$ month intervals. Follow up included examination of wound and healing assessment, measurement of the ankle / brachial pressure indices, handheld arterial doppler and duplex scanning of the treated limb. If indicated, CT angiography was ordered and performed and patients were rescheduled back after the scan was done.

- Immediate hemodynamic improvement: Post procedural ABI improvement by 0.15 as compared to pre-procedural baseline. Detected in $48 / 54$ cases $(88.8 \%)$ and not in 6 cases (11.1\%).

- Sustained hemodynamic improvement: Sustained ABI improvement of 0.15 through follow up. In case of successful first endovascular procedure. Detected in 34 cases $(70.8 \%)$ and not in 10 cases $(29.1 \%)$.

\section{- Sustained clinical improvement:} Considered as cumulative clinical improvement of a single class in case of claudication, or two classes in case of tissue loss according to Rutherford classification with or without minimal hemodynamic improvement of an ABI of 0.15 without need for re-intervention. Alternatively, sustained clinical improvement is considered with healing of ulcer and / or disappearance of rest pain at 3 months. This was detected in $34 / 48$ cases $(70.8 \%)$ and not in 14 cases (29.1\%).

- Binary restenosis (BR): peak velocity ratio of 2.4 of target lesion or $50 \%$ re obstruction of target lesion) and time to binary restenosis. Detected in $11 / 54$ cases (22.9\%) and not in 37 cases $(77.1 \%)$. Time to BR is (mean; 49.43 week), (SD; 53.174).

- Target lesion revascularization (TLR): frequency for need for redo procedure (endovascular or open) after successful first procedure due to stenosis involving the SAME lesion, and time to TLR. Detected in 14/48 cases $(29.1 \%)$ and not in 34 cases $(70.8 \%)$. Time to TLR is (mean; 35 week), (SD; 40.229).

- TER (target extremity revascularization): frequency for need for redo-procedure (endovascular or open) due to stenosis or 
occlusion in the same limb, but a DIFFERENT lesion. Detected in 2/54 cases (3.7\%) and not in 52 cases $(96.4 \%)$.

- Minor amputation. Detected in 13/54 cases $(24.5 \%)$ and not in 40 cases $(75.5 \%)$, Time to minor amputation was (mean; 4 weeks), (SD; 6.831).

- Major amputation (proximal to ankle). Detected in only one case (1.9\%). Time to Major amputation (mean; 2 weeks).

- Limb salvage (no need for major amputation). Detected in 52/54 cases (98.1\%) and not in 2 cases (1.9\%) The limb salvage was done by trial of endovascular procedures or surgical bypass (assertion to treatment).

- No mortality was detected within $\mathbf{3 0}$ days, nor Major complication (Defined as complications occurring in the perioperative period that required re-operation or a extension of the hospital stay for at least 24 hours).

- Primary patency: primary patency of endovascular intervention within period of 2 years was $34 / 48$ cases $(70.9 \%)$.

- Assisted primary patency (the durability of an intervention that failed initially due to re-stenosis, but did not reach the level of thrombosis, and was consequently retreated) was $73.33 \%$.

- Secondary patency (Secondary patency means failure of the initial intervention to the level of thrombosis and was re-treated. Once the second intervention was successfully accomplished, secondary patency was defined as the durability of that second intervention) was $68.18 \%$.

- Drug eluting balloon has lower incidence of restenosis and need for intervention (BR, TLR) in comparison to standard balloon (10\% vs $29.4 \%$, $10 \%$ vs $42.4 \%$, respectively). Stent insertion was associated with high incidence of re-intervention (TLR) in comparison to balloon only angioplasty (68.4\% vs $31.6 \%)$.

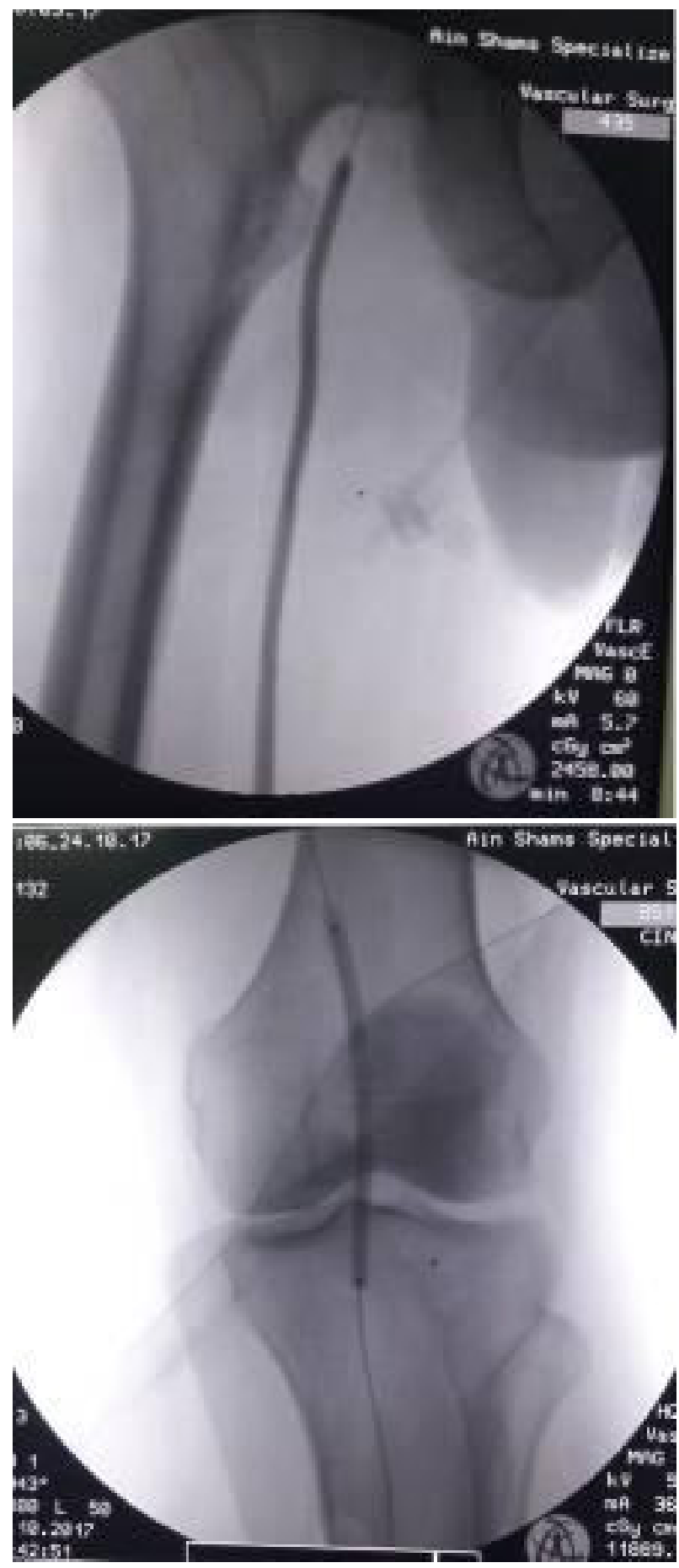

Fig 1: SFA and Popliteal Balloon angioplasty.

\section{Discussion}

Open bypass VS endovascular management, Our conviction is still that bypass surgery is better adopted for long SFA occlusive lesions of more than $25 \mathrm{~cm}$ length, using an autologous vein graft, provided that the patient's life expectancy is more than 2 years. Endovascular treatment is an attractive alternative to open surgical procedures for PAD. We didn't compare between open and endovascular intervention for TASC II D femorpopliteal classification. It's single arm study on surgical high risk patients ASA grade 3 and 4 .

Primary SFA stent VS. angioplasty-alone CTO SFA 
lesion, The RESILIENT study (Randomized study comparing the Edwards Self-expanding Lifestent vs. angioplasty-alone In Lesions Involving the SFA and/ or proximal popliteal artery) presented 3 years follow up. It reported preference for primary stenting. The freedom from target lesion revascularization after 1 year ( 3 years) in the stent group was $87.3 \%$ (75.5\%) vs. $45.1 \%$ (41.8\%) for balloon angioplasty. In the RESILIENT study all the lesions were $<15-\mathrm{cm}$ long and the patients were claudicants. ${ }^{8}$

In our study, we adopted angioplasty alone strategy + /- stent insertion if needed, not primary stenting. Stenting was only used in cases of unsatisfactory hemodynamics on angiography. This is usually due to elastic recoil, significant stenosis of $>30 \%$ or flow limiting dissection. Stent insertion was in 28/48 cases $(58.33 \%)$ while $19 / 48$ cases didn't needed stents $(39.58 \%)$, stent insertion was associated with high incidence of re-intervention and TLR in comparison to balloon only angioplasty (68.4\% vs $31.6 \%$ ). This is against results of previous mentioned studies. However, low number of cases for significance, and the gradual onset of symptoms for balloon only strategy group and use only bare metal stents make the comparison not accurate.

Spotstenting VS. long stenting in long femoropopliteal complete total occlusion: Long stenting was identified as an independent predictor of restenosis. Theoretically, longer lesion length, increased fracture rate, small artery or stent diameter, or the stentto-artery diameter mismatch may lead to a higher restenosis rate in the long stenting group than in the spot stenting group. However, we found no significant differences regarding these variables. ${ }^{9}$ In our study, When stenting was required, we stented the entire subintimal channel including entry and re-entry points with a self-expanding stents with the shortest possible lengths. When we were in need to use more than one stent, the stents were overlapping without skip lesions.

Role of Drug eluting balloons DEBs: Drug-eluting balloons provide a safe technology and it has been demonstrated that even the short-term exposure to paclitaxel-coated balloon catheters is sufficient to inhibit restenosis. DEB's have been shown to consistently reduce restenosis rates and the need for revascularization in the SFA in several independent randomized clinical trials, with low bail-out stenting rates. ${ }^{10}$

We used in this study 3 types of Balloons, standard (36/54; 72\%), High pressure $(3 / 54 ; 6 \%)$ and DEBs (11/54; 22\%). Low number of used high pressure balloon make its consideration insignificant. Drug eluting balloon has less incidence of binary restenosis in comparison to standard balloon $(10 \%$ vs. $29.4 \%$ ) and need of re-intervention (target limb revascularisation) (10\% vs. $42.4 \%)$.

\section{Conclusion}

Endovascular management has an effective role in treatment of TASC II D femo-popliteal occlusive diseases in surgical high risk patient. Technical success reported in $88.9 \%$, The Primary patency within 2 years is $70.9 \%$, Primary assisted patency is $73.33 \%$ and Secondary patency is $68.18 \%$.

Drug eluting balloons have lower incidence of restenosis and need for in comparison to standard balloon. Stent insertion is associated with high incidence of re-intervention (TLR) in comparison to balloon only angioplasty .

\section{Reference}

1. Robertson L, Paraskevas KI, Stewart M: Angioplasty and stenting for peripheral arterial disease of the lower limbs: An overview of Cochrane Reviews. Cochrane Database of Systematic Reviews. https://doi. org/10.1002/14651858.CD012542. 2017.

2. McDermott $M$, Greenland $P$, Liu K, Guralnik J, Criqui M, Dolan N, et al: Leg symptoms in peripheral arterial disease: associated clinical characteristics and functional impairment. JAMA. 2001; 286(13): 1599-1606.

3. Uccioli L, Meloni M, Izzo V, Giurato L, Merolla S, Gandini R: Critical limb ischemia: Current challenges and future prospects. Vascular Health and Risk Management. 2018; 14, 63-74. https://doi.org/10.2147/VHRM.S125065.

4. Norgren L, Pande RL, Preux PM, Stoffers HE: Treat-Jacobson D. Measurement and interpretation of the ankle-brachial index: A scientific statement from the American Heart Association. Circulation. 2012; 126: 28902909.

5. Bosiers M, Peeters P, Tessarek J, Deloose K, Strickler S: The Zilver PTX single-arm study investigators. The Zilver_ PTX_ single arm study: 12-month results from the TASC C/D lesion subgroup. J Cardiovasc Surg (Torino). 2013; 54:115-122.

6. Adam DJ, Beard JD, Cleveland T, Bell J, Bradbury AW, Forbes JF: Bypass versus angioplasty in severe ischaemia of the leg (BASIL): Multicentre, randomised controlled trial. Lancet. 2005; 366: 1925-1934.

7. Bradbury AW, Adam DJ, Bell J, Forbes JF, Fowkes FGR, Gillespie I, et al: Bypass versus angioplasty in severe ischaemia of the leg (BASIL) trial: An intention-to-treat analysis of amputation-free 
and overall survival in patients randomized to a bypass surgery-first or a balloon angioplastyfirst revascularization strategy. J Vasc Surg. 2010; 51 (Suppl 10): 5S-17S.

8. Laird JR, Katzen BT, Scheinert D, et al: Nitinol stent implantation vs. balloon angioplasty for lesions in the superficial femoral and proximal popliteal arteries of patients with claudication: three-year follow-up from the RESILIENT randomized trial. J Endovasc Ther. 2012; 19: 1-9.
9. Hong SJ, Ko YG, Shin DH, Kim JS, Kim BK, Choi D, Hong MK, Jang Y: Outcomes of spot stenting versus long stenting after intentional subintimal approach for long chronic total occlusions of the femoropopliteal artery. JACC Cardiovasc Interv. 2015; 8(3): 472-480.

10. Van den Berg JC: Drug-eluting balloons for treatment of SFA and popliteal disease-a review of current status, European Journal of Radiology. 2017; 91: 106-115. 